\title{
Adaptive optics imaging of Lyman break galaxies as progenitors of spheroids in the local Universe $\dagger$
}

\author{
M. Akiyama ${ }^{1}$, Y. Minowa ${ }^{2}$, N. Kobayashi ${ }^{3}$, K. Ohta ${ }^{4}$ and I. Iwata \\ ${ }^{1}$ Subaru Telescope, National Astronomical Observatory of Japan, 650 North A'ohoku Place, \\ Hilo, HI, 96720, USA \\ email: akiyama@naoj.org \\ ${ }^{2}$ Optical and Infrared Astronomy Division, NAOJ, Mitaka, 181-8588, Japan \\ ${ }^{3}$ Institute of Astronomy, University of Tokyo, Mitaka, 181-0015, Japan \\ ${ }^{4}$ Department of Astronomy, Kyoto University, Kyoto 606-8502, Japan \\ ${ }^{5}$ Okayama Astrophysical Observatory, NAOJ, Okayama 719-0232, Japan
}

\begin{abstract}
In order to reveal the stellar mass distribution of $z \sim 3$ galaxies, we are conducting deep imaging observations of U-dropout Lyman Break Galaxies (LBGs) with Adaptive Optics (AO) systems in $K$-band, which corresponds to rest-frame $V$-band of $z \sim 3$ galaxies. The results of the Subaru intensive-program observations with AO36/NGS/IRCS indicate that 1) the Kband peaks of some of the LBGs brighter than $K=22.0$ mag show significant offset from those in the optical images, 2) the $z \sim 3 M_{V}^{*}$ LBGs and serendipitously observed Distant Red Galaxies (DRGs) have flat profiles similar to disk galaxies in the local universe (i.e., Sérsic with $n<2$ ), and 3) the surface stellar mass densities of the $M_{V}^{*}$ LBGs are 3-6 times larger than those of disk galaxies at $z=0-1$. Considering the lack of $n>2$ systems among the luminous $z \sim 3$ LBGs and DRGs, and their strong spatial clustering, we infer that the dense $n<2$ disk-like structures evolve into the $n>2$ spheroids of nearby galaxies through relaxations due to major merger events.
\end{abstract}

Keywords. galaxies: formation, galaxies: high-redshift, cosmology: observations

\section{Introduction}

The statistical evolution of the morphology of galaxies is one of the most fundamental observational clues to understand the establishing process of the Hubble sequence of galaxies seen in the local universe. Especially rest-frame optical morphology is important because it reflects stellar mass distribution of galaxies. High-resolution imaging observations with Hubble Space Telescope (HST), covering rest-frame optical wavelength up to $z \sim 1$, have shown that the Hubble sequence is already established at $z \sim 1$. Morphological studies on galaxies at higher redshifts started following the discovery of a large number of high redshift galaxies using Lyman Break selection (Steidel et al. 2003). HST NICMOS observations of the Hubble Deep Field North (HDFN) show that the morphology of the LBGs is essentially independent of the wavelength up to the rest-frame $4000 \AA$ (Giavalisco 2002).

However, the NICMOS observations are not sufficient to conclude the distribution of the stellar mass in $z \sim 3$ LBGs. The NICMOS observations covers up to the $H$-band,

$\dagger$ Based on data collected at Subaru Telescope, which is operated by the National Astronomical Observatory of Japan. 

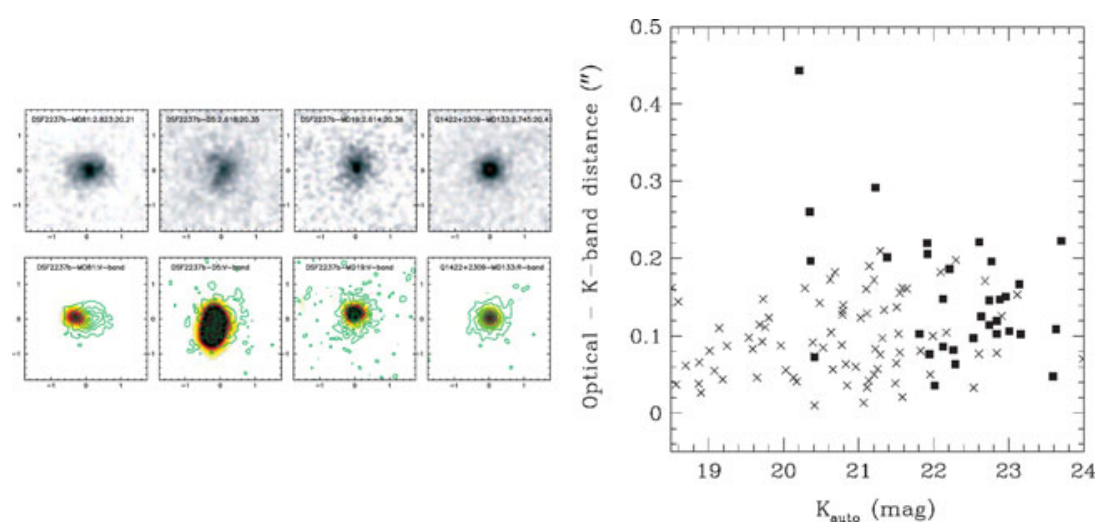

Figure 1. Left) $K$-band images (upper) and optical images with $K$-band contour (lower) of the 4 brightest $z \sim 3$ LBGs in the sample. Each panel has a $3 .^{\prime \prime} 5 \times 3 .^{\prime \prime} 5(27 \mathrm{kpc} \times 27 \mathrm{kpc}$ at $z=3)$ FoV. North is to the top and east is to the left. We do not match the PSF size of the $K$-band contour to that of the optical images, thus only the positions of the peaks should be compared. Right) Distance between $K$-band and optical peaks as a function of $K$-band magnitudes for the $z \sim 3$ LBGs (filled squares) and field object (crosses).

which is just at $4000 \AA$ in the rest-frame of $z \sim 3$ galaxies. In addition, the NICMOS observations are mostly limited to the $z \sim 3$ LBGs in the small HDFN area and only include object as bright as $M_{B} \sim-22 \mathrm{mag}$, which is still $0.5 \mathrm{mag}$ fainter than the characteristic absolute magnitude of the $z \sim 3$ LBGs $\left(M_{V}^{*}=-24.0 \mathrm{mag}\right)$.

In order to examine the rest-frame $V$-band morphology of the $z \sim 3$ LBGs covering a wide luminosity range $\left(M_{V}^{*}-0.5 \mathrm{mag}-M_{V}^{*}+3.0 \mathrm{mag}\right)$, we conducted AO-assisted imaging observations of 36 of them in the $K$-band, which corresponds to the rest-frame $V$-band in $z \sim 3$ galaxies (Akiyama et al. 2007). Thirty one of the LBGs are detected in the deep imaging observations with typical effective integration time of 5 hours.

\section{Results of AO imaging observations of the $z \sim 3$ LBGs}

The AO-assisted $K$-band images of the 4 brightest $z \sim 3$ LBGs are shown in the upper left panel of Figure 1 as examples. The AO observations clearly resolve most of the 31 $z \sim 3$ LBGs at the resolution of FWHM $\sim 0 .{ }^{\prime \prime} 2$.

We examined their total $J-K$ colors, which corresponds to the rest-frame $U-V$ colors, using seeing-limited $J$-band data of thirty of the LBGs. The bright LBGs show red rest-frame $U-V$ colors (average of $0.2 \mathrm{mag}$ ), while most of the fainter LBGs show blue rest-frame $U-V$ color (average of $-0.4 \mathrm{mag}$ ). The color distribution of the LBGs show that the LBG sample covers not only blue less massive galaxies but also red massive galaxies at among $2<z<3$ galaxies selected with photometric redshifts.

In the lower left panels of Figure 1 , the $K$-band contours of the LBGs are compared with the optical images with the seeing-limited resolution. The peaks in the $K$-band images of the LBGs show significant offset from those in the optical images. In the right panel of Figure 1, the shifts of the LBGs are shown as a function of $K$-band magnitude with filled squares for the whole sample. In order to evaluate the uncertainties of the shift measurements, we also plot the differences of $K$-band and optical positions of compact field objects with crosses. The peaks in the $K$-band images of 7 of the LBGs with $K<22$ mag show significant or marginal shifts from those in the optical images. The presence 

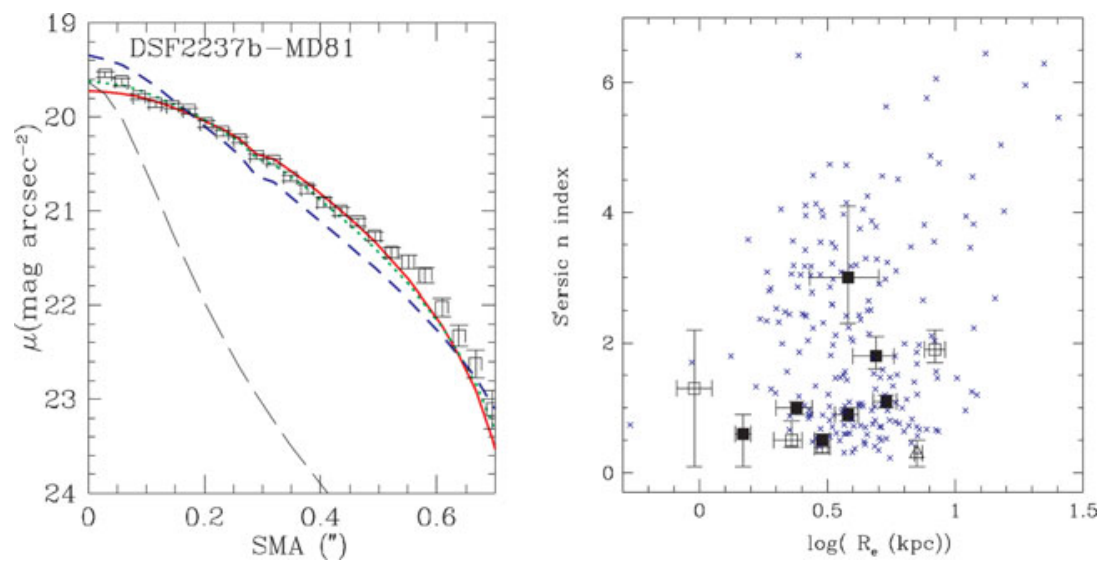

Figure 2. Left) $K$-band profiles of an LBG along the semi-major axis. The red-solid, bluedashed, and green-dotted lines show the best fit Sérsic (with free $n$ ), $r^{1 / 4}$ (Sérsic with $n=4$ ), and exponential (Sérsic with $n=1$ ) profiles, respectively. Long dashed line show the estimated profiles of the PSFs at the object positions. Right) $R_{e}$ vs. $n$ for the $z \sim 3$ LBGs (filled squares) and DRGs (open squares) with $K<21.5$ mag. Simulated $z=3$ galaxies that are brighter than $K=21.5 \mathrm{mag}$ in the $2 \mathrm{mag}$ PLE model are plotted with blue small crosses.

of the shifts among red luminous LBGs implies that the UV-bright star-forming regions are not necessarily centered at their main body observed in the $K$-band.

In order to examine whether their light profiles are similar to those of local galaxies or not, we apply one component Sérsic profile fitting to the AO-assisted $K$-band images of the LBGs with $K<21.5 \mathrm{mag}$. An example of the Sérsic profile fitting is shown in the left panel of Figure 2 (DSF2237b-MD81 with $K=20.2 \mathrm{mag}$ at $z=2.82$ ). As can be seen in the panel, the exponential profile (Sérsic profile with $n=1$ ) fit the profile better than the $r^{1 / 4}$ profile (with $n=4$ ), and the best-fit $n$ is 0.9 with free $n$ fitting. The results of the Sérsic profile fitting of the LBGs with $K<21.5 \mathrm{mag}$ are shown in the right panel of Figure 2 with filled and open squares, respectively. We also examined the profiles of serendipitously observed Distant Red Galaxies in the FoVs. The images of all but one of the LBGs and DRGs with $K<21.5$ mag are fitted well with Sérsic profile with $n$ index less than 2, similar to disk galaxies in the local universe.

In order to directly compare the best-fit parameters with those of galaxies at intermediate redshifts, we make simulated images of $z \sim 3$ galaxies by "cloning" HST/ACS images of galaxies at $z=0.4-0.7$. Their distribution is shown with blue crosses in the panel. The LBGs and DRGs locate similar part of the $R_{e}$ vs. $n$ plane to the disk galaxies at intermediate redshifts.

Assuming that the $z \sim 3$ LBGs and DRGs have a disk shape, we compare their sizeluminosity and size-stellar mass relations with those of disk galaxies at low/intermediate redshifts in the left and right panels of Figure 3, respectively. It should be noted that still there is a possibility that the $z \sim 3$ LBGs and DRGs have a spheroidal shape with $n=1$ profile instead of the disk shape. The $z \sim 3$ LBGs are brighter than $z=0$ and 1 disk galaxies at the same $R_{e}$. The surface brightness of the LBGs, which are estimated from $M_{V}$ and $R_{e}$, are 2.2-2.9 mag and 1.2-1.9 mag brighter than those of the disk galaxies at $z=0$ and 1 , respectively. The size-stellar mass relation indicates that the $z \sim 3$ LBGs brighter than $M_{V}^{*}$ have the average surface stellar mass density 3-6 times larger than those of the $z=0$ and 1 disk galaxies. On the contrary, for less-luminous $z \sim 3$ LBGs, their size-stellar mass relation is similar to those of $z=0$ and 1 disk galaxies. 

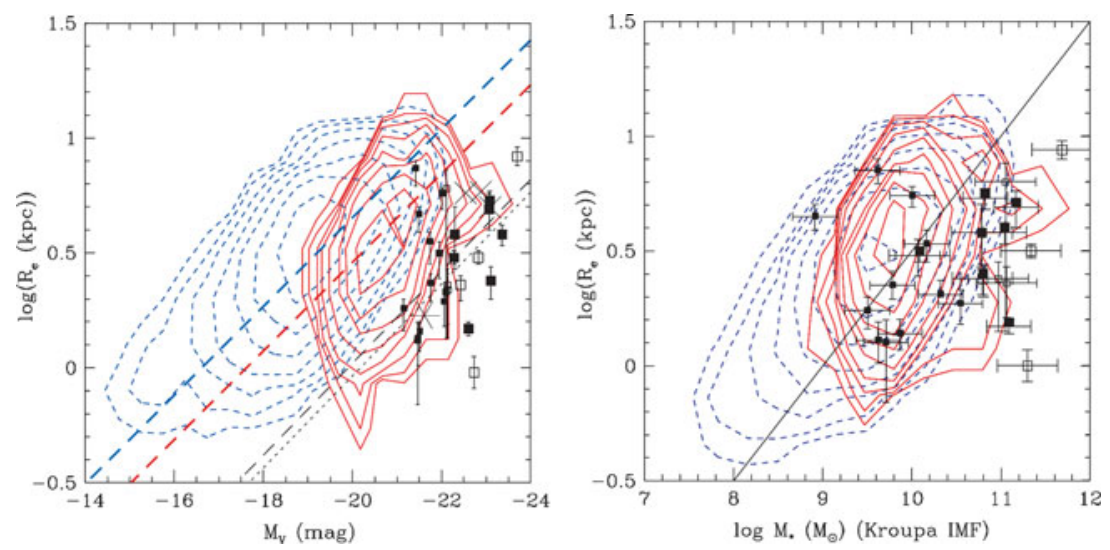

Figure 3. Left) $M_{V}$ vs. $R_{e}$ for the LBG sample (filled squares). Large and small symbols indicate $R_{e}$ obtained with the Sérsic profile fitting with free $n$ and fixed $n=1$, respectively. Open squares show the DRG sample. Contours show the distributions of $z=0$ (dashed blue contour) and $z=1$ (solid red contour) disk-galaxies from Barden et al. (2005). The blue and red dashed lines show $\left\langle\mu_{V}>\right.$ of disk galaxies at $z=0\left(20.84 \mathrm{mag} \operatorname{arcsec}^{-2}\right)$ and $z=1(19.84 \mathrm{mag}$ $\operatorname{arcsec}^{-2}$ ), respectively. Right) $M_{*}$ vs. $R_{e}$ of the LBG (filled squares) and DRG (open squares) samples. Contours show the distributions of $z=0$ and 1 disk-galaxies from Barden et al. (2005). The solid line represents the relation $\log \Sigma_{M}\left(M_{\odot} \mathrm{kpc}^{-2}\right)=8.50$ derived from the disk galaxies at $z=0-1$ (Barden et al. 2005).

\section{Speculation: LBGs before $z \sim 3$ and after}

Because in the local universe, such disk with high surface stellar mass density as the massive $z \sim 3$ LBGs is rare, we expect that the disks of the massive $z \sim 3$ LBGs are destroyed between $z \sim 3$ to 1 . The strong spatial clustering of the LBGs implies that they reside in massive dark halos and that they evolve into local spheroids (Adelberger et al. 2005). Therefore, the disks with high surface stellar mass density would evolve into local spheroids through "dry" merging events.

Considering the difference between the mass of the dark matter halos the $z \sim 3$ LBGs reside $\left(2-6 \times 10^{11} M_{\odot}\right.$; Adelberger et al. 2005) and the Jeans mass at the time of reionization $\left(\sim 10^{10} M_{\odot}\right)$, we naively expect that the $z \sim 3$ LBGs form from builing blocks through several major merges. The high fraction of disk-like galaxies in the highredshift universe would be explained with a hypothesis that they have been going through only gas-rich "wet" merges at that time (e.g., Springel \& Hernquist 2005).

We start an AO observing program with laser guide stars in order to extend the sample of bright $z \sim 3$ galaxies, and to establish the morphological evolution from $z=3$ to 0 . The high-resolution $K$-band imaging observations of the galaxies at $z \geqslant 3$ should be one of the unique fields that can be explored only with LGS AO on ground-based 8-10m class telescopes until the launch of the James Webb Space Telescope.

\section{References}

Adelberger, K. L., Steidel, C., Pettini, M., Shapley, A. E., Reddy, N. A., \& Erb, D. K. 2005, ApJ 619, 697

Akiyama, M., Minowa, Y., Kobayashi, N., Ohta, K., Ando, M., \& Iwata, I. 2007, ApJS submitted

Barden, M., et al. 2005, ApJ 635, 959

Giavalisco, M. 2002, ARA\&A 40, 579

Springel, V. \& Hernquist, L. 2005, ApJ 622, L9

Steidel, C., et al. 2003, ApJ 592, 728 